\title{
prolessional directory
}

Space reserved for professional members only. For rates apply to:

Executive Director, AMERICAN METEOROLOGICAL SOCIETY, 45 Beacon St., Boston, Mass. 02108

\section{A. H. GLENN AND ASSOCIATES}

ALFRED H. GLENN

\section{Certified Consulting Meteorologist}

Consultants in Meteorology and Oceanography

Registered Engineers in Louisiana and Texas

New Orleans Lakefront Airport, New Orleans, La. 70126:

504-241-2222

3927 Essex Lane, Houston, Tex. 77027: 713-621-3500

Cable Address: GLENN, New Orleans

NORTHEAST WEATHER SERVICE, INC.

JOHN E. WALLACE

JOHN P. MURPHY

Certified Consulting Meteorologists

Specializing in Operational Forecasts

131A Great Road

Bedford, Massachusetts 01730

617-275-8860 TWX 710-326-0799

\section{WEATHER CORPORATION OF AMERICA}

Consulting Professional Meteorologists

With Hundreds of Successful Case Histories ... Weather Modification ... Advertising \& Marketing ... Gas \& Electric Utili-

ties ... Radio and Television ... Design and Construction ...

Legal and Insurance . . . Government Projects . . . Shipping . . . Aviation.

611 Olive Street, St. Louis 1, Missouri

GArfield 1-5566 TWX: 314-556-0010

Cable: Weather, Saint Louis

NORTH AMERICAN

WEATHER CONSULTANTS

\section{AEROMETRIC}

RESEARCH

INC.

ROBERT D. ELLIOT'T

EINAR L. HOVIND

Certified Consulting Meteorologists

Weather Modification, Environmental and Air Pollution

Field Studies, Weather and Marine Forecasting, Clima-

tology, and Applied Research.

805-967-1246

Teletype: 910-334-1181

Santa Barbara Municipal Airport, Goleta, Calif. 93017

METEOROLOGY RESEARCH, INC.

Atmospheric Physics Research - Weather Modification Programs • Diffusion Studies - Air Pollution Surveys - Field Investigations • Instrumentation

464 West Woodbury Rd., Altadena, Calif. 91001

P.O. Box 637

213-791-1901
MURRAY AND TRETTEL, INCORPORATED

JOHN R. MURRAY

DENNIS W. TRETTEL

Certified Consulting Meteorologists

Operational Forecasts, Environmental Studies, Forensic Meteorology, Research Programs.

414 West Frontage Road, Northfield, Illinois 60093

312-446-7800

WEATHER SERVICES, INC.

A Subsidiary of Northeast Weather Service

PETER R. LEAVITT

\section{Certified Consulting Meteorologist}

Specializing in Pollution Studies, Statistical Research, Forensic Meteorology and Expert Testimony

131 A Great Road

Bedford, Massachusetts 01730

617-275-8862 TWX 710-321-0305

LEO ALPERT, Ph.D.

Certified Consulting Meteorologist

4120 34th Street North

Arlington, Virginia 22207

$703-524-3145$

WALLACE E. HOWELL, Sc.D.

Certified Consulting Meteorologist

RFD 3 Box 400

Golden, Colorado 80401

303-277-9125

\section{ALLEN WEATHER CORPORATION}

Meteorologists-Oceanographers

4461 Connecticut Ave. N. W.

Washington 8, D. C.

EMerson 3-7221

Cable: ALWEX WADC 
Space reserved for professional members only. For rates apply to:

Executive Director, AMERICAN METEOROLOGICAL SOCIETY, 45 Beacon St., Boston, Mass. 02108

\section{DeNARDO \& McFARLAND}

Weather Services, Inc.

Municipal, Industrial and Aviation Forecasts; Atmospheric and Climatological Research Utilizing Computer Facilities

TWX 710-794-4804 412-462-6464

Allegheny County Airport, West Mifflin, Pennsylvania 15122

\section{NATIONAL WEATHER CORPORATION} WORLD-WIDE WEATHER SERVICES

Forecasting-Flight Planning-Data Analysis-Surveys-Displays

Hangar Twelve, Newark Airport, Newark, N.J. 07114 201-624-8110

Hangar Seven International Airport

Los Angeles, Calif. 90045

Hangar D

Westchester County Airport

213-776-5507

White Plains, N.Y. 10601

914-428-6500

\section{HENRY T. HARRISON}

\section{Certified Consulting Meteorologist}

Reems Creek

Route 1 Box 266

Weaverville, North Carolina 28787

704-645-3706

\section{ATMOSPHERICS INCORPORATED}

Operational Weather Modification and Cloud Physics Research Weather Radar and Meteorological Systems Applications Airborne and Ground Based Particulate Measurements

4981 East Dakota, Fresno, California 93727 209-291-4161

$E G \& G, I N C$.

Laboratory \& Field Services

Standard \& Research Instrumentation

933 Bradbury Drive, S.E.

Albuquerque, New Mexico 87106

505-842-4000

\section{WEATHER ENGINEERING CORPORATION} OF CANADA, LTD.

P.O. Box 2060,

315 Dorval Avenue,

Dorval 780, Quebec, Canada.

514-636-0293

\section{GCA CORPORATION \\ GCA TECHNOLOGY DIVISION}

Consultants to industry and government in air pollution assessment, measurement, and control.

Bedford, Massachusetts 01730

Salt Lake City, Utah 84115 617-275-9000 801-487-1607

VINCENT J. SCHAEFER, Sc.D.

\section{Certified Consulting Meteorologist}

Research Consultant

R. D. 3, Box 36, Schermerhorn Road

Schenectady, New York 12306

EXpress 3-8978

518-393-8978

\section{METEOROLOCY INTERNATIONAL} INCORPORATED

Technologies in the Analysis and Prediction of Physically and Operationally Significant Variabilities in the Atmosphere and Oceans, Satellite Meteorology, Circulation Dynamics, Computer Systems Applications

P. O. Box 349

419 Webster Street, Monterey, California 93940 408-372-5173

\section{ALLIED RESEARCH ASSOCIATES, INC.}

Research and Operational Services in-Atmospheric and

Environmental Sciences; Geophysical Satellite Data Utilization; and Remote Sensing of Earth Resources

617-369-9000

Virginia Road, Concord, Massachusetts 01742 


\section{prótessional direetiory}

Space reserved for professional members only. For rates apply to:

Executive Director, AMERICAN METEOROLOGICAL SOCIETY, 45 Beacon St., Boston, Mass. 02108

\section{THE WALTER A. BOHAN COMPANY}

WALTER A. BOHAN, DIRECTOR

Certified Consulting Meteorologist

Applied Meteorological Research

Specialized Studies and Surveys

2026 Oakton Street, Park Ridge, Illinois 60068

312-825-3677

WEATHER TRENDS INTERNATIONAL, LTD.

Specializing in Long-Range Forecasts, Weather/Marketing Research \& Promotional Weather Services in North America and Europe

565 Fifth Ave., N.Y., N.Y. 10017 (212) MU 2-4280

European HQ: London

SOUTHEAST WEATHER CONSULTANTS, INC.

Consultants For Research, Aviation \& Industry

AVMETS *

Specializing in Programming Jet Minimum Cost Cruise Control Flight Planning For Air Carriers and Business Jets

Southern Aero Building P.O. Box 20637

Atlanta Airport Branch, Atlanta, Georgia 30320

404-761-6991

* Aviation Meteorological Service

LOREN W. CROW

\section{Certified Consulting Meteorologist}

2422 South Downing Street

Denver, Colorado 80210

303-722-8665

or 756-3971

\section{METRONICS ASSOCIATES, INC.}

W. A. PERKINS, PRESIDENT P. A. LEIGHTON, BD. CHMN. S. W. GRINNELL, F. X. WEBSTER, L. M. VAUGHAN

Environmental Services ... Atmospheric Research

FP Air Tracer Systems . . . Rotorod Samplers

Stanford Industrial Park

415-326-5632
3201 Porter Drive

Palo Alto, California 94304
INTERNATIONAL WEATHER CORPORATION WALTER F. ZELTMANN, PRESIDENT

Consultant Meteorologists ... Oceanography

Forecasts ... Statistical Surveys .. Legal and Insurance

P. O. Box 176, Bay Ridge Station, Brooklyn, N. Y. 11220

212-854-0300

WEATHER SCIENCE, INC

D. RAY BOOKER, PRESIDENT

Environmental Data Systems

Environmental Data Services

Weather Modification

P.O. Box FF

Norman, Oklahoma 73069

405-329-5263 or 329-7007

\section{ERWIN K. KAUPER}

\section{Certified Consulting Meteorologist}

Associated with Pollution Research \& Control Corp.

616 E. Colorado St., Glendale, Calif. 91205

213-240-0515

\section{CENERAL WEATHER CENTER}

Directed by Lyle E. Brosché.

Effective Long and Short Range Specialized Forecasts, and Consulting, for Business and Industry. Excellent Client References.

915 Fisher Building, Detroit, Michigan 48202

313-871-3511 TWX 810-221-1287

\section{E. BREWSTER BUXTON}

\section{Certified Consulting Meteorologist}

Aviation-Engineering-Research

Box 322, Congress Street

Morrisville, Vermont 05661

802-888-4566 


\section{protessional direclopy}

Space reserved for professional members only. For rates apply to:

Executive Director, AMERICAN METEOROLOGICAL SOCIETY, 45 Beacon St., Boston, Mass. 02108

\section{WEATHER SURVEYS}

A Div. of National Weather Corp.

CONRAD B. GOSSET, General Manager

Specializing in investigations for

Law Firms and Insurance Companies

34 Nannahagen Road, Pleasantville, N.Y. 10570

914-769-1350

Branch Offices: Albany, N.Y., Hartford, Conn., New Haven, Conn., Los Angeles, Calif.,

Newark, N.J. and Washington, D.C.

\section{BERNARD H. DeWITT \& ASSOCIATES}

\section{Consultants in Meteorology \& Air Safety}

2109 Windsor Dr.

Ann Arbor, Michigan 48103

313-769-4944

313-485-2300

\section{GULF COAST WEATHER SERVICE}

Commercial Section of

WTVT Television Weather Service

Serving individuals and industry since 1961

Roy Leep

Director

Tampa, Florida

813-876-3592

\section{EASTERN AIRLINES}

Meteorology Department

J. J. GEORGE, DIRECTOR

Certified Consulting Meteorologist

General Meteorology Practice-Aviation-Applied ResearchSpecial Studies-Forecasting

Miami International Airport

Miami, Florida 33148

305-873-2681

\section{SIERRA RESEARCH CORPORATION}

\section{Environmental Systems Group}

RALPH PAPANIA

\section{Certified Consulting Meteorologist}

Weather Modification and Fog Dispersal

Air Pollution Monitoring \& Analysis, Environmental Consulting

Weather Radar, Instrumentation, Automatic Data Systems

P. O. Box 3007, Boulder, Colorado 80303

303-443-0384
SIDNEY R. FRANK . . GROUP

SIDNEY R. FRANK, PRESIDENT

\section{Certified Consulting Meteorologist}

General Consulting for Industry and Government

Environmental Research \& Development Programs

Pollution Analyses-Theoretical and Operational

1500 Cecil Cook Place

FAA Building

Santa Barbara Municipal Airport 805-964-4477

Goleta, Calif. 93017

Home: 805-965-7540

\section{BENDIX COMMERCIAL SERVICE CORP.}

Marine Science Services - Optimum Ship Routing - Ocean

Weather Forecasts - Marine Operations Consulting

Teterboro, New Jersey 07608

212-947-9000 or 201-288-1550

Cable BEXMARSVC, TTBR

WORLD WEATHER, INC.

Warm Fog Dispersal and General

Modification Services Worldwide

620 Commercial Tower

P. O. Box 22250

Midland, Texas 79701

Sacramento, Calif. 95822

916-422-6791

E. R. A. SERVICES, INC.

RICHARD E. CALE, PRESIDENT

Certified Consulting Meteorologist

Environmental Research Applications

Aviation Weather Investigations

Weather Prediction Services

P.O. Box 352, Cerritos, California 90701

714-956-0220

Special Weather Services

Division-Columbia Research Corp.

CHARLES GERTZ, METEOROLOGICAL DIRECTOR

Specialists: Weather Modification, Ship Routing, Marine Forecasts, Aviation and Industry, Climatological studies. All services domestic-at sea-abroad

P. O. Box 485, Gaithersburg, Md. 20760

301-948-2450 


\section{Drolessional direectory}

Space reserved for professional members only. For rates apply to:

Executive Director, AMERICAN METEOROLOGICAL SOCIETY, 45 Beacon St., Boston, Mass. 02108

\section{R. ROBERT RAPP, Ph.D.}

\section{Certified Consulting Meteorologist}

49 Uplifters Ranch

Santa Monica, California 90402

213-454-8878

\section{INSTITUTE FOR STORM RESEARCH}

at the University of St. Thomas

JOHN C. FREEMAN, DIRECTOR

\section{Certified Consulting Meteorologist}

Research in theoretical and applied meteorology, oceanography, mathematics

4104 Mt. Vernon, Houston, Texas 77006

713-529-4891 Telex 762-771

OCEANROUTES, INC.

JOHN D. PLOETZ

\section{Certified Consulting Meteorologist}

Consultants in Meteorology and Oceanography

Operational Forecasts for the Marine Industry

Optimum Ship Routing

1534 Page Mill Road

Palo Alto, California 94304

TWX TELEX

910-373-1175 345-540

Stanford Industrial Park

$\begin{array}{ll}\text { CABLE } & \text { TELEPHONE } \\ 415-493-3600\end{array}$

\section{ENVIRONMENTAL WEATHER SERVICE}

A Service of WFLA, Inc.

Legal - Insurance - Pollution Research - Aviation Industry •

Agricultural Forecast - Marine

P. O. Box 1410

Tampa, Florida 33601

813-229-7781
Paul Catoe

George Wooten

Bill Seiler
C. EUGENE BUELL, Ph.D., P.E.

\section{Certified Consulting Meteorologist}

Kaman Sciences Corporation

1700 Garden of the Gods Road

Colorado Springs, Colo., 80907

$303-473-5880$

\section{DAMES \& MOORE}

R. G. ALLEN - H. B. VISSCHER - 404-875-9971 - ATLANTA, GA. IRWIN SPICKLER · 202-833-9430 - WASHINGTON, D.C.

J. W. HATHORN · 312-297-6120 - PARK RIDGE, ILL.

CONSULTING ENGINEERS IN THE APPLIED EARTH SCIENCES

Services in Meteorology

Diffusion Studies - Regional Planning for Air Quality - Air Quality Monitoring - Atmospheric Heat Sink Studies - Nuclear Industry Safety Reports - Industrial Effects on Air Quality

Offices in Principal Cities Around the World

AEROVIRONMENT INC.

PAUL B. MAC CREADY JR., PH.D., PRESIDENT

\section{Certified Consulting Meteorologist}

Consulting and programs in weather modification, air pollution, instrumentation, aircraft systems and operations.

P. O. Box 4525

Pasadena, California 91106

213-449-4392

CONTROL DATA CORPORATION

Meteorology Department, Research Division CONSULTING SERVICES

Assessment of air pollution potential; diffusion modelling - Design of air quality sampling and meteorological networks - Site surveys - Climatological and statistical analyses - Mesoscale measurements with "METRAC" - Data processing systems

612-853-3595

P.O. Box 1249

Minneapolis, Minnesota 55440

Certified Consulting Meteorologist: The certification program of the American Meteorological Society is aimed to foster the establishment and maintenance of a high level of professional competency, and mature and ethical counsel, in the fields of consulting meteorology. Requirements of knowledge, experience and character are determined by a five-man Board. Objectives of the program in full detail and applications may be obtained from the headquarters of the Society, 45 Beacon Street, Boston, Mass. 02108. 